\title{
Pentraxin 3 (PTX3) inhibits plasma cell/stromal cell cross-talk in the bone marrow of multiple myeloma patients
}

\author{
Antonio Basile,'† Michele Moschetta,'† Paolo Ditonno, ${ }^{2}$ Roberto Ria,' Ilaria Marech,' Annunziata De Luisi,' \\ Simona Berardi,' Maria Antonia Frassanito,' Emanuele Angelucci, ${ }^{3}$ Daniele Derudas, ${ }^{3}$ Giorgina Specchia, ${ }^{4}$ \\ Paola Curci, ${ }^{4}$ Vincenzo Pavone, ${ }^{5}$ Bernardo Rossini, ${ }^{5}$ Domenico Ribatti, ${ }^{6}$ Barbara Bottazzi, ${ }^{7}$ Alberto Mantovani, 7,8 \\ Marco Presta, ${ }^{*}$ Franco Dammacco' and Angelo Vacca!* \\ I Department of Biomedical Sciences and Human Oncology, University of Bari Medical School, Bari, Italy \\ 2 Haematology Unit, Ospedale 'Di Venere', Bari, Italy \\ 3 Department of Haematology, Ospedale Businco, Cagliari, Italy \\ ${ }^{4}$ Haematology and Transplantation Unit, Department of Emergency and Organ Transplantation, University of Bari Medical School, Bari, Italy \\ 5 Haematology Unit, Fondazione dell'Ospedale Cardinale G Panico, Tricase, Italy \\ 6 Department of Human Anatomy, Histology and Embryology, University of Bari Medical School, Bari, Italy \\ 7 Istituto Clinico Humanitas Mirasole, University of Milan, Rozzano, Milan, Italy \\ ${ }^{8}$ Department of Translational Medicine, University of Milan, Rozzano, Milan, Italy \\ 9 Unit of General Pathology and Immunology, Department of Biomedical Sciences and Biotechnology, University of Brescia Medical School, \\ Brescia, Italy
}

*Correspondence to: Professor Angelo Vacca, MD, PhD, Department of Biomedical Sciences and Human Oncology (DIMO), Section of Internal Medicine and Clinical Oncology, Policlinico, Piazza Giulio Cesare II, I-70I 24 Bari, BA, Italy. e-mail: a.vacca@dimo.uniba.it

*Correspondence to: Professor Marco Presta, MD, PhD, General Pathology and Immunology, Department of Biomedical Sciences and Biotechnology, School of Medicine, University of Brescia, Viale Europa I I, I-25 I 23 Brescia, Italy. e-mail: presta@med.unibs.it

tThese authors contributed equally to the work.

\begin{abstract}
Pentraxin 3 (PTX3) is a soluble pattern recognition receptor that binds with high affinity and selectivity to fibroblast growth factor-2 (FGF2), thus inhibiting its pro-angiogenic activity. Here we investigated the effects of PTX3 on monoclonal gammopathy of undetermined significance (MGUS) and multiple myeloma (MM) patientderived bone marrow (BM) plasma cells (PCs), endothelial cells (ECs), and fibroblasts (FBs), and assessed whether PTX3 can modulate the cross-talk between PCs and those microenvironment cells. PTX3 and FGF2 expression was evaluated by ELISA. Functional studies, including cell viability, wound healing, chemotaxis, and Matrigel ${ }^{\circledR}$ assays, were performed on MGUS and MM ECs and FBs upon the PTX3 treatment. Through western blot PTX3-induced modulation in FGF2/FGF receptor signalling pathways was evaluated in MGUS and MM ECs and FBs through western blot. Co-cultures between MM ECs/FBs and human PC lines were used to evaluate possible PTX3 indirect effects on MM PCs. Adhesion molecules were studied by flow cytometry. PTX3 provides a direct time- and dose-dependent apoptotic effect on MM ECs and FBs, but not on either MM primary PCs or human PC lines. PTX3 inhibits migration of MM ECs and FBs in a dose-dependent manner, and impacts in vitro and in vivo FGF2-mediated MM angiogenesis. Co-cultures of PCs and ECs/FBs show that PTX3 treatment indirectly impairs PC viability and adhesion. We conclude that PTX3 is an anti-angiogenic factor in MM and behaves as a cytotoxic molecule on MM cells by inhibiting the cross-talk between PCs and ECs/FBs.

Copyright $\odot 2012$ Pathological Society of Great Britain and Ireland. Published by John Wiley \& Sons, Ltd.
\end{abstract}

Keywords: pentraxin 3; multiple myeloma; MGUS; angiogenesis; bone marrow microenvironment; FGF2

Received 9 May 2012; Revised 27 June 2012; Accepted 22 July 2012

No conflicts of interest were declared.

\section{Introduction}

Multiple myeloma (MM) remains an incurable disease, despite conventional and high-dose chemotherapies [1]. Molecules targeting not only plasma cells (PCs) but also bone marrow (BM) stromal cells (BMSCs) such as endothelial cells (ECs) and fibroblasts (FBs) are needed to overcome drug resistance [2]. Angiogenesis is a constant component of the MM microenvironment
[3]. It results from a prevalence of activatory over inhibitory interactions between PCs and BMSCs, and ultimately guides the survival and drug resistance of PCs [4]. It takes place with the 'angiogenic switch' in step with transition from the avascular phase of the monoclonal gammopathy of undetermined significance (MGUS) to the vascular phase of active MM [3]. On the switch, pro-angiogenic factors overcome the anti-angiogenic ones [5], and fibroblast growth 
factor-2 (FGF2), a heparin-binding protein, plays a pivotal role among the former [6]. It acts as both an autocrine and a paracrine factor on PCs, ECs, and FBs [7], being a potent mitogen for these cells, and inducing their migration and differentiation [7-9]. Pentraxin 3 (PTX3), a 41-kDa glycosylated protein assembled in 10-20 mer multimers [10], is a prototypic member of the long pentraxin subfamily [11], a soluble pattern recognition receptor, and an anti-angiogenic factor $[12,13]$. Its unique $\mathrm{N}$-terminal domain contains a (97-110) amino acid sequence that binds FGF2 with high affinity and selectivity [14,15]. This prevents FGF2 binding to cognate receptors (FGFRs) and heparan-sulphate proteoglycans, as demonstrated in human and bovine normal ECs [8], thus halting the FGF2 pro-angiogenic activity $[8,16]$. Accordingly, PTX3 inhibits EC proliferation and migration, and FGF2-dependent neovascularization in chick embryo chorioallantoic membrane (CAM) [15]. PTX3 is produced in various physiopathological conditions by ECs and FBs $[17,18]$.

Here we wondered whether PTX3 plays a role in the modulation of $\mathrm{EC}$ and $\mathrm{FB}$ activities that lead to MM-associated angiogenesis and of PC viability and adhesion to those stromal cells. By studying how PTX3 interferes with the cross-talk between the stromal cells and PCs, we demonstrate that FGF2 is more expressed than PTX3 in the BM microenvironment of MM versus MGUS and control subjects, and that PTX3 inhibits EC and FB activities and PC adhesion to these cells in MM patients and behaves as an indirect cytotoxic molecule on PCs. Our observations point to PTX3 as a potential anti-angiogenic factor that could be employed to reestablish a physiological FGF2/PTX3 ratio in the MM microenvironment.

\section{Materials and methods}

\section{Patients and controls}

The BM aspirates from patients fulfilling the International Myeloma Working Group diagnostic criteria for active MM $(n=35)$ and MGUS $(n=24)$ were studied. MM patients (20 males and 15 females) were aged 47-86 (median 63.5) years and D\&S staged as IIA $(n=8)$, IIB $(n=6)$, IIIA $(n=16)$, and IIIB $(n=5)$; the M-component was $\operatorname{IgG}(n=20), \operatorname{IgA}(n=9)$, and $\kappa$ or $\lambda(n=6)$. MGUS patients (14 males and 10 females) were aged 41-80 (median 61.5) years and were $\operatorname{IgG}(n=12), \operatorname{IgA}(n=8)$ or $\kappa$ or $\lambda(n=4)$. Normal (control) ECs and FBs were derived from 14 subjects with anaemia due to iron or vitamin B12 deficiency (age range: 48-79 years; median: 62.8 years) [19]. The study was approved by the Ethics Committee of the University of Bari Medical School and all patients gave their informed consent in accordance with the Declaration of Helsinki.

\section{Cell cultures, plasma, and conditioned media}

BM mononuclear cells were harvested from aspirates by Ficoll gradient. ECs, FBs, and PCs were isolated with specific magnetic microbeads as described previously [4]. Conditioned media (CM) were prepared as described previously [20]. Plasma was separated from $\mathrm{BM}$ aspirates. Methods are described in detail in the Supplementary materials and methods.

\section{Reagents}

Recombinant human PTX3 (provided by Dr Bottazzi) was dissolved in PBS, pH 7.0 and used at 33-198 $\mathrm{nM}$ in in vitro assays and at 0.11 and $0.22 \mathrm{nM}$ in the in vivo CAM assay as previously assessed [15]. Recombinant human FGF2 and VEGF (Peprotech, London, UK) were dissolved at $0.1 \mathrm{M}$ in $5 \mathrm{mM}$ Tris, pH 7.6 with $150 \mathrm{mM} \mathrm{NaCl}$ and used at 0.58 and 0.26 $\mathrm{nM}$, respectively.

\section{Functional studies}

Viability, wound healing, chemotaxis, Matrigel ${ }^{\circledR}$, chick embryo chorioallantoic membrane (CAM), and adhesion assays were performed as previously described [21-24] and are described in detail in the Supplementary materials and methods.

\section{Adhesion molecules analysis}

Plasma cell lines RPMI8226 and U266 (both at $5 \times 10^{5}$ per dish) and MM ECs and FBs (both at $2.5 \times 10^{4}$ per dish) were seeded in $3.5 \mathrm{~cm}^{2}$ dishes with SFM alone or supplemented with $0.58 \mathrm{nM}$ FGF2 alone or with 66 and $132 \mathrm{nM}$ PTX3. After $24 \mathrm{~h}$, cells were analysed (CD11a, CD54, CD49d, CD49e, CD29) by a FACSCanto II cytofluorimeter (BD Biosciences, San José, CA, USA) using the FlowJo software (Tree Star, Ashland, OR, USA). Methods are described in detail in the Supplementary materials and methods.

\section{Western blotting and ELISA}

Protein lysates $(30 \mu \mathrm{g})$ from MM ECs and FBs, seeded in SFM supplemented with $0.58 \mathrm{nM} \mathrm{FGF2} \mathrm{and} \mathrm{PTX3}$ $(0,66$, and $132 \mathrm{nM})$ for $15 \mathrm{~min}$, were separated on 4-12\% NuPAGE Novex Bis-Tris gels (Invitrogen, Carlsbad, CA, USA) [21]. Primary antibodies to Akt, phospho(p)-Akt, Src, p-Src, Erk1/2, p-Erk1/2, NFкB, p-NFкB (Cell Signaling Technology, Denvers, MA, USA), and anti-tubulin antibody (as loading control; Cell Signaling Technology) were used. NFKB and pNFKB were analysed in MM ECs and FBs seeded in SFM supplemented with 0.58 nM FGF2 and PTX3 (0, 66 , and $132 \mathrm{nM}$ ) for $25 \mathrm{~min}$ and then treated with TNF- $\alpha$ (10 ng/ml) for $10 \mathrm{~min}$, as previously described [21]. The secreted PTX3 and FGF2 were quantified in CM and BM plasma by an ELISA (R\&D Systems, Minneapolis, MN, USA). 


\section{Co-cultures of MM ECs and FBs with PC lines}

MM ECs and FBs (both at $2.5 \times 10^{4}$ per dish) from five active MM patients were co-cultured in 24-well plates with paired MM PCs or RPMI8226 or U266 cells $\left(5 \times 10^{4}\right.$ per well), with or without transwell insert, and treated with 66 and $132 \mathrm{nM}$ PTX3. The PC lines were previously marked with Carboxyfluorescein $\mathrm{N}$ succinimidyl ester (CFSE) to discriminate PCs from the adherent ECs and FBs. Apoptosis of MM ECs, FBs, and PCs was determined using PE Annexin V/7Amino-actinomycin D (7-AAD; BD Biosciences) and the FACSCanto II cytofluorimeter.

\section{Results}

PTX3 and FGF2 expression in bone marrow PCs, ECS, and FBs, and plasma of MM versus MGUS patients and control subjects

First, we measured FGF2 and PTX3 levels in CM of all cytotypes and found their significant increase in ECs and FBs of MM versus MGUS patients and control subjects (Figures 1A and 1B). Since angiogenesis results as a balance between pro- and anti-angiogenic factors, data were analysed as FGF2/PTX3 ratio. According to their pro-angiogenic state, MM patients showed a ratio significantly higher than that of MGUS patients (Figure 1C): indeed, it was increased in MM
PCs, ECs, and FBs compared with the MGUS counterparts. FGF2 and PTX3 levels were also assessed in BM plasma. As shown in Figure 1D, the FGF2/PTX3 ratio was higher in MM than in MGUS. Notably, control subjects overlapped MGUS patients (Figure 1D).

\section{PTX3 impacts FGF2-dependent activities of MM ECs and FBs}

Based on the above evidence, the effect of PTX3 on FGF2-dependent EC (Figure 2A) and FB (Figure 2B) functional activities was investigated next. ECs and FBs from MM and MGUS patients and from control subjects were stimulated with $0.58 \mathrm{nM} \mathrm{FGF2}$ and treated with increasing doses of PTX3. The viability of FGF2-exposed MM ECs and FBs was dosedependently affected by PTX3: at 24 h, 66 and 132 nM PTX3 reduced MM EC viability by $24 \%$ and $41 \%$, respectively, and maximal inhibition (55\%) was obtained at $198 \mathrm{nM}$ (Figure 2A). Similar effects were observed on $\mathrm{FB}$ viability, with maximal inhibition (41\%) again at $198 \mathrm{nM}$ (Figure 2B). These effects were absent or marginal in MGUS ECs and FBs, as well as in cells isolated from control subjects (Supplementary Figures 1 and 2). Of note, normal ECs (HUVECs) and normal FBs (WI-38) overlapped controls (data not shown). MM ECs and FBs treated with just PTX3 but not FGF2 displayed only a marginal inhibitory effect ( $-21 \%$ and $-20 \%$ at $198 \mathrm{nM}$, respectively), due to the seizure of only endogenous FGF2 (data not shown).

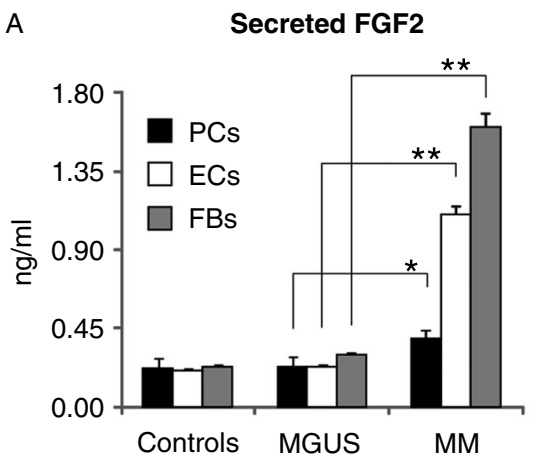

B

Secreted PTX3
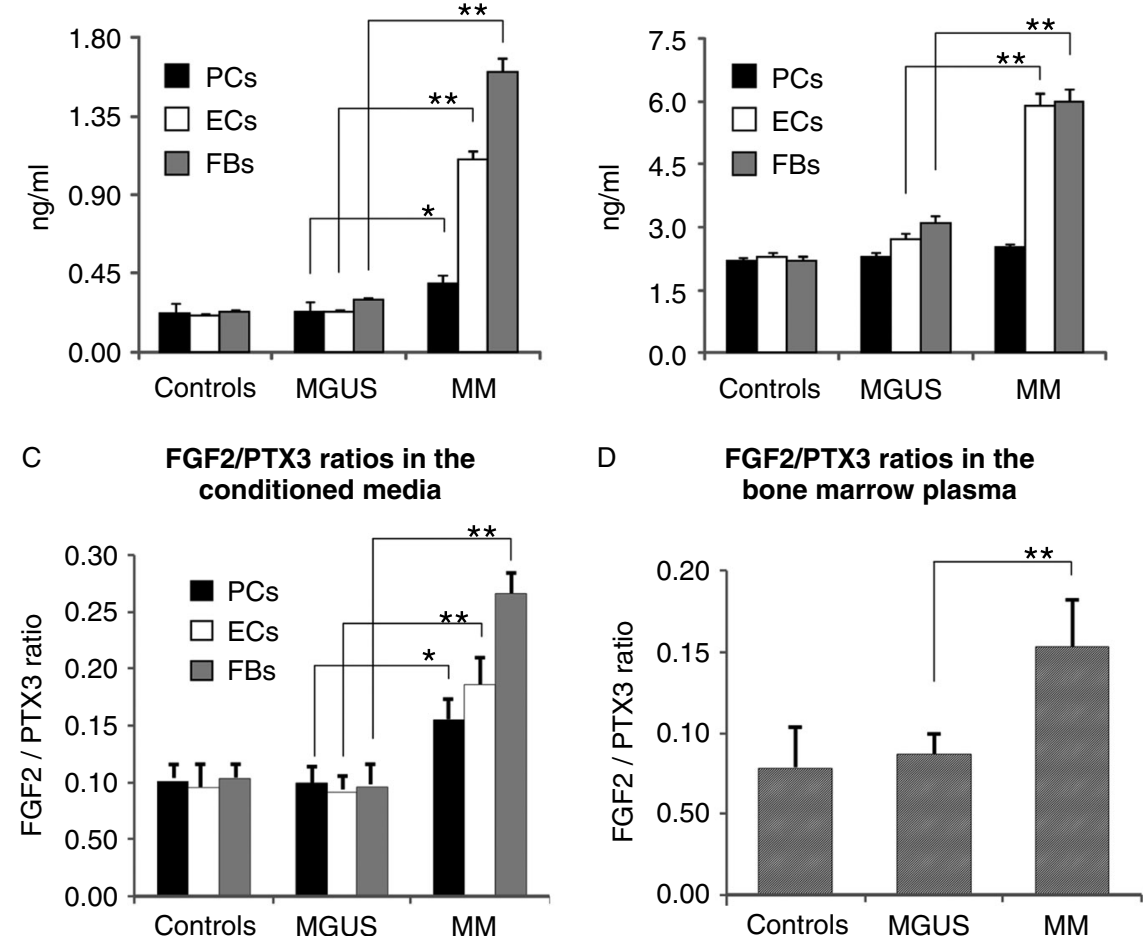

Figure 1. FGF2 and PTX3 production in PC, EC, and FB conditioned media and in BM plasma. FGF2 (A) and PTX3 (B) levels in the conditioned media of plasma cells (PCs), endothelial cells (ECs), and fibroblasts (FBs) as well as in BM plasma of 14 control subjects, 24 MGUS, and 35 active MM patients were examined by ELISA. Values are expressed as mean \pm standard deviation. FGF2/PTX3 ratios were calculated in conditioned medium (C) and BM plasma (D). ${ }^{*} p<0.03,{ }^{* *} p<0.01$; Wilcoxon signed-rank test. 

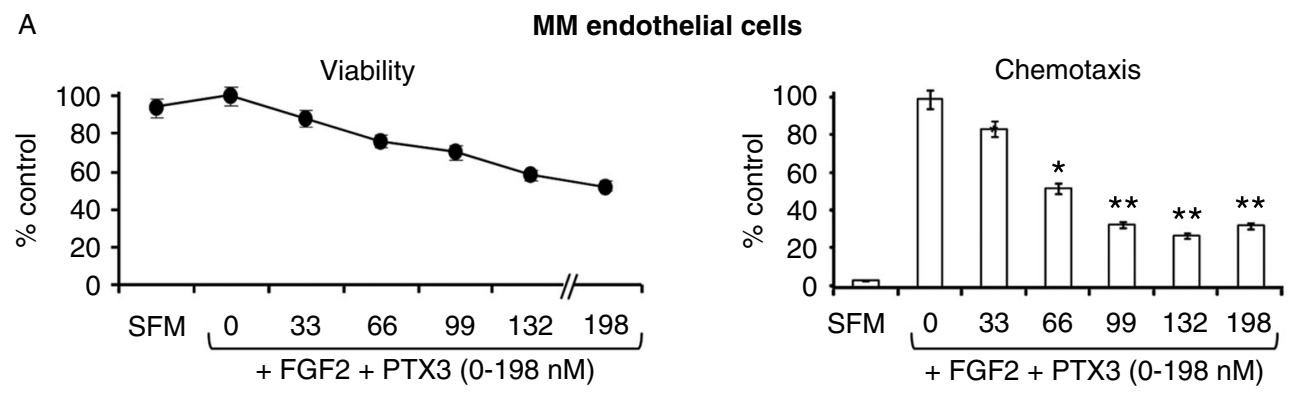

Wound Healing

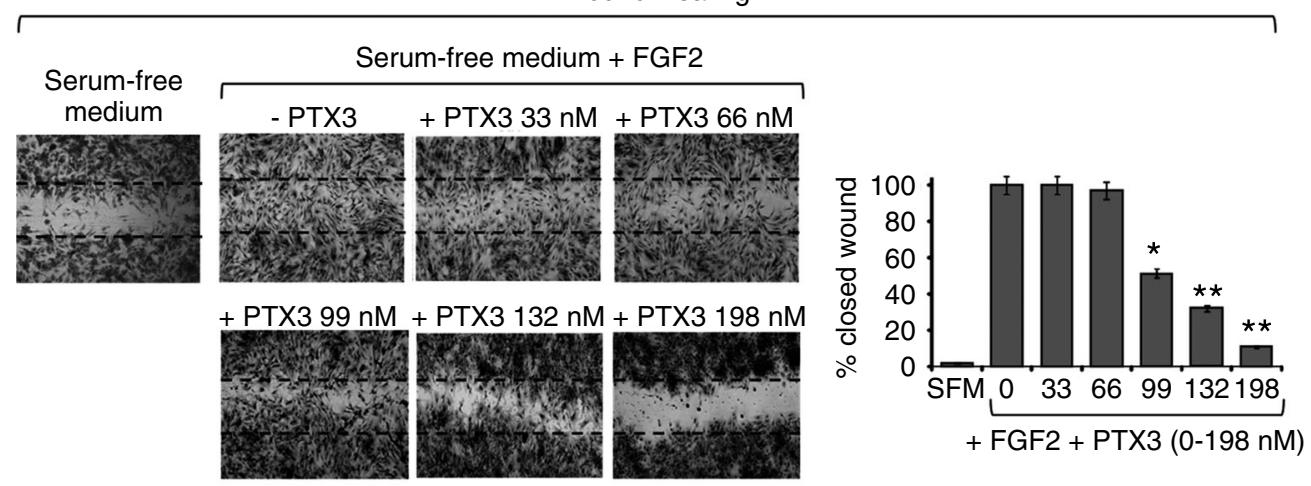

B

MM fibroblasts
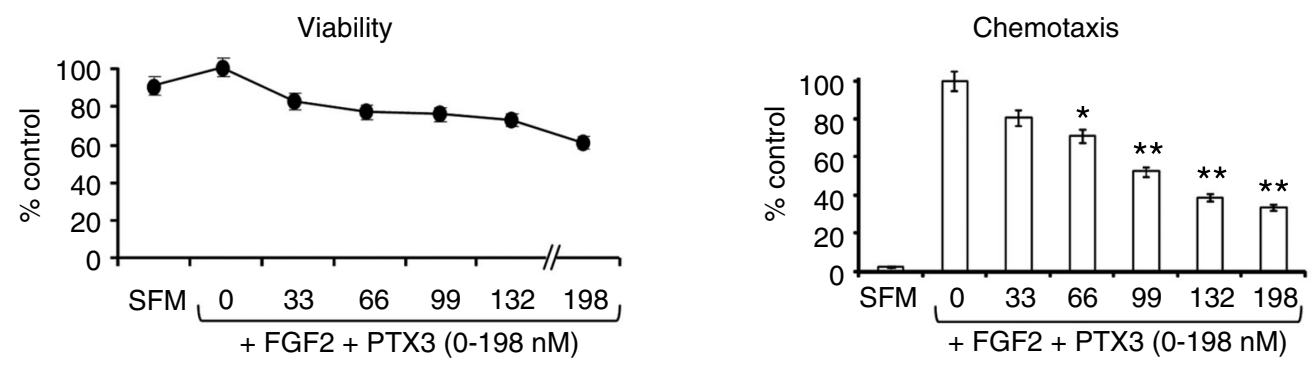

Wound Healing

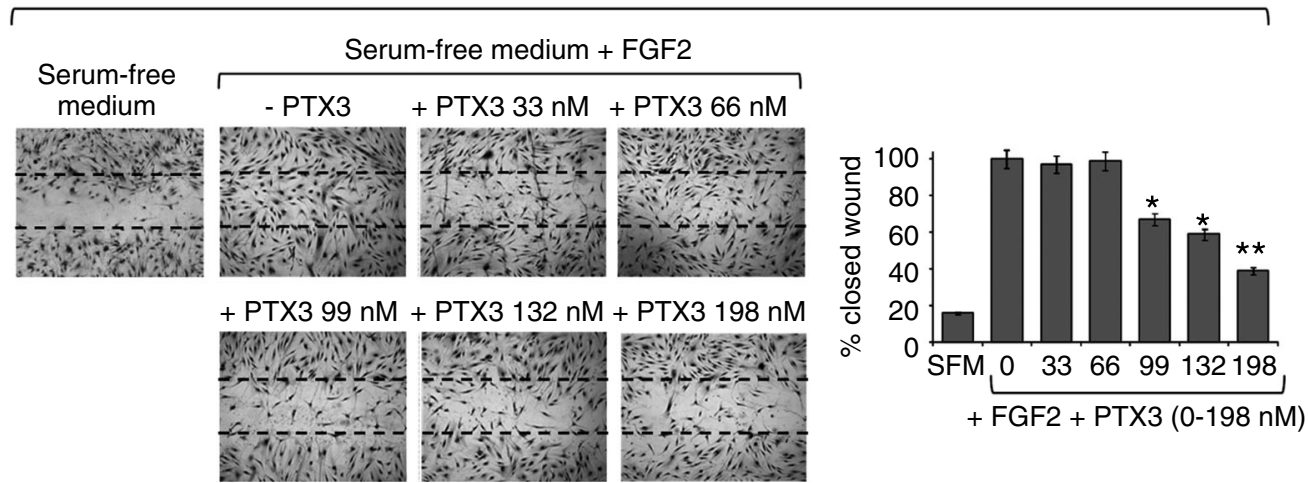

Figure 2. PTX3 affects MM endothelial cell (EC) and fibroblast (FB) functional activities. Viability, chemotaxis, and wound healing assays were performed on ECs (A) and FBs (B) isolated from the BM of $27 \mathrm{MM}$ patients and cultured in triplicate in serum-free medium (SFM) and SFM supplemented with FGF2 $(0.58 \mathrm{nM})$ and PTX3 doses $(0,33,66,99,132$, and $198 \mathrm{nM})$. Wound healing assays from a representative patient are shown. ${ }^{*} p<0.03,{ }^{* *} p<0.01$; Wilcoxon signed-rank test.

PTX3 also inhibited EC and FB migration in a dosedependent manner in MM but not in MGUS patients, as assessed by a chemotaxis (directional migration) and a wound (random migration) assay. Chemotaxis of MM ECs was inhibited by $50 \%$ and $72 \%$ (Figure 2A), and that of FBs by $29 \%$ and $61 \%$ (Figure 2B), at 66 and $132 \mathrm{nM}$ PTX3, respectively. In the corresponding MGUS cells and in control subjects' ECs and FBs, the inhibitory effect was only marginal (Supplementary Figures 1 and 2). Migration to heal the wound was impacted in a dose-dependent fashion by PTX3 on MM ECs (Figure 2A) and FBs (Figure 2B). These effects were again marginal in MGUS ECs and FBs, as well as in control subjects' cells (Supplementary Figures 1 and 2). HUVECs and WI-38 cells behaved like the corresponding MGUS and control cells (data 


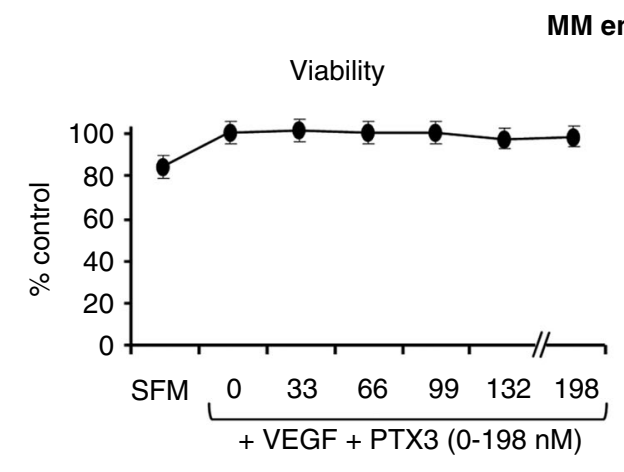

\section{M endothelial cells}
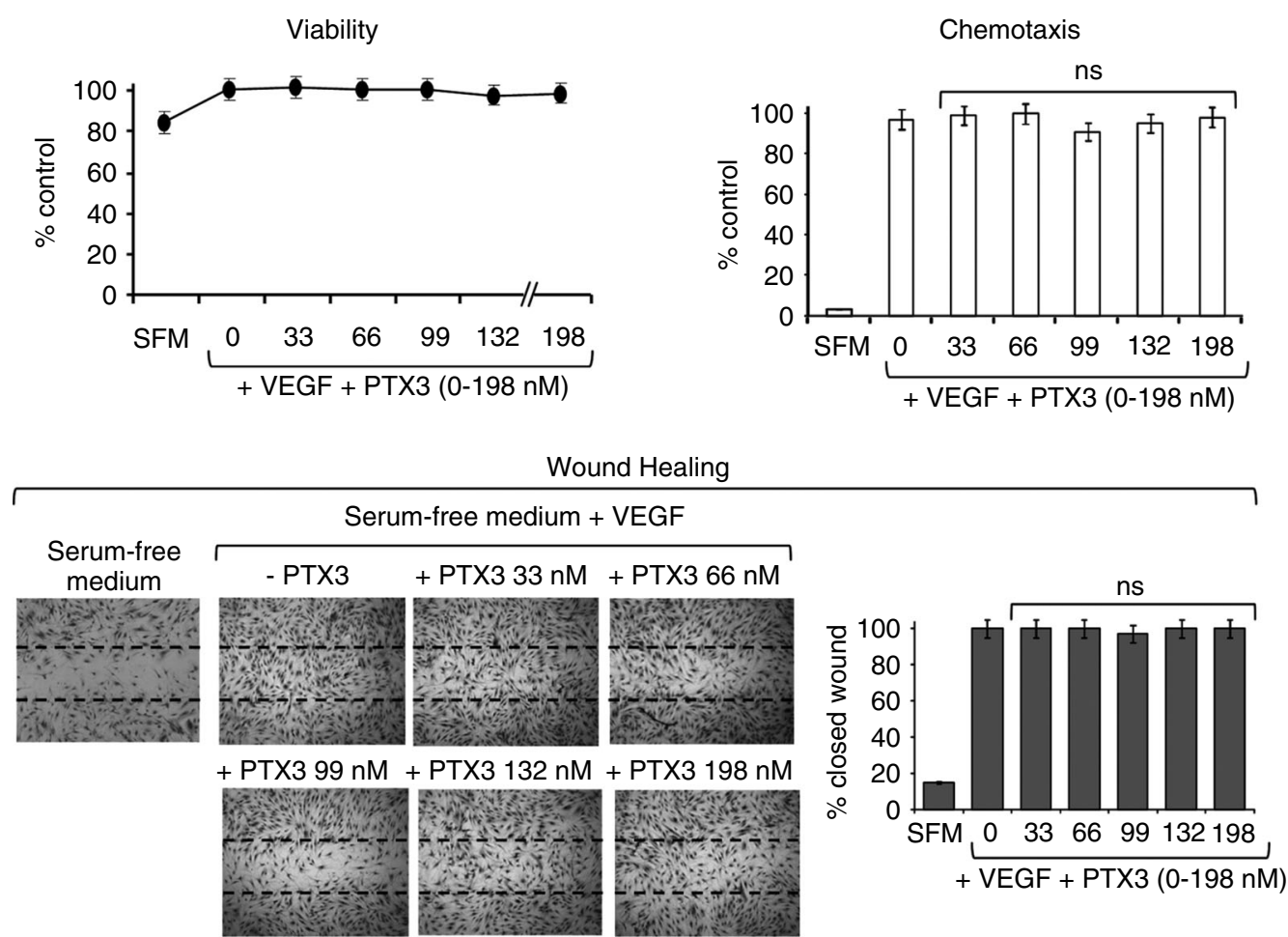

Figure 3. PTX3 does not affect VEGF-dependent functional activities of MM endothelial cells (ECs). Viability, chemotaxis, and wound healing assays were performed on ECs isolated from the BM of $27 \mathrm{MM}$ patients and cultured in triplicate in serum-free medium (SFM) and SFM supplemented with VEGF $(0.26 \mathrm{nM})$ and PTX3 doses $(0,33,66,99,132$, and $198 \mathrm{nM})$. Wound healing assays from a representative patient are shown. $\mathrm{ns}=$ not significant; Wilcoxon signed-rank test.

not shown). When cells were exposed to VEGF instead of FGF2, PTX3 was not able to antagonize viability and migratory activity exerted by VEGF on MM ECs (Figure 3), indicating that PTX3 specifically affects FGF2-dependent cell functions.

\section{PTX3 inhibits FGF2-dependent angiogenesis in MM}

On the Matrigel $^{\circledR}$ in vitro angiogenesis assay, PTX3 caused dose-dependent inhibition of vessel mesh areas, length, and branching points on FGF2-stimulated MM ECs (Figure 4A), but not in stimulated control subjects' and MGUS ECs (Supplementary Figure 3). Furthermore, the inhibitory effect of PTX3 on angiogenesis was also analysed by the in vivo CAM assay [15]. Indeed, PTX3 was able to inhibit angiogenesis induced by the CM of primary MM PCs (which contains FGF2) [25] by reducing vessel counts by $42 \%$ and $77 \%$ at 0.11 and $0.22 \mathrm{nM}$ doses, respectively (Figure 4B). Overall, the data indicate that PTX3 is able to inhibit FGF2dependent neovascularization in MM.

\section{PTX3 down-regulates the FGF2 signalling and} cytokine secretion in MM ECs and FBs

Major signalling effector molecules in FGF2 pathways such as phospho (p)-Akt, p-Erk1/2, p-Src, and p-NFKB were examined in MM ECs and FBs upon PTX3 exposure. At $66 \mathrm{~nm}$, PTX3 p-Src levels decreased by $22 \%$ in MM FBs, while p-Erk1/2 levels decreased by $20 \%$ in MM ECs (Figures 5A and 5B). At $132 \mathrm{~nm}$,
PTX3 p-Akt and p-Src levels were respectively reduced by $37 \%$ and $26 \%$ in MM ECs, and by $38 \%$ and $53 \%$ in MM FBs (Figures $5 \mathrm{~A}$ and $5 \mathrm{~B}$ ). A $47 \%$ reduction of $\mathrm{p}$ Erk1/2 was observed in MM ECs upon 132 nM PTX3 exposure. The p-NFkB levels were reduced by $50 \%$ and $61 \%$ in MM ECs, and by $37 \%$ and $53 \%$ in MM FBs at 66 and 132 nM PTX3, respectively (Figures 5A and $5 \mathrm{~B})$. These data indicate that PTX3 significantly prevents the activation of FGF2 downstream pathways implicated in MM ECs and FB viability and migration.

PTX3 was next investigated for possible effects on MM ECs' and FBs' secretion of the stromal cell derived factor-1 alpha (SDF-1 $\alpha$ ), interleukin-8 (IL8), interleukin-6 (IL-6), and monocyte chemotactic protein-1 (MCP-1), which are all involved in MM progression [26,27]. As shown in Figure 5C, SDF-1 $\alpha$ secretion was reduced by $26 \%$ and $40 \%$ in $\mathrm{CM}$ of $\mathrm{MM}$ ECs, and by $49 \%$ and $61 \%$ in CM of MM FBs at 66 and 132 nM PTX3, respectively. IL-8 was lowered by $22 \%$ and $33 \%$ in CM of MM ECs, and by $27 \%$ and $36 \%$ in CM of MM FBs at the same above doses. Finally, MCP-1 was decreased by $20 \%$ and $27 \%$ in PTX3-treated MM ECs.

\section{PTX3 impacts MM PC viability and adhesion}

Since the MM BM microenvironment is the source of an intricate network of signals (via adhesion molecules and cytokines) which support PC viability, proliferation, and migration, we wondered whether PTX3 


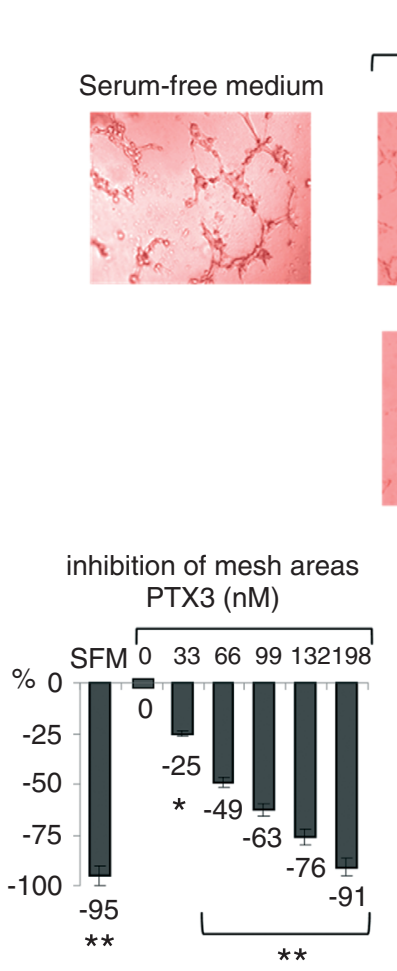

B

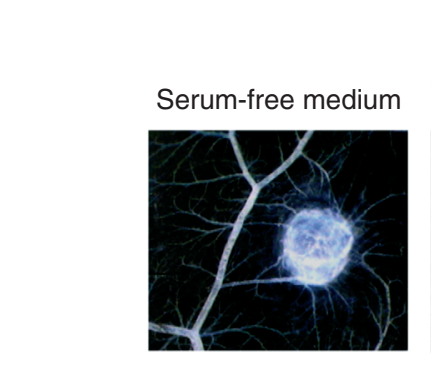

Vessel counts $\quad$ n. $=7 \pm 2$
Matrigel assay on MM endothelial cells

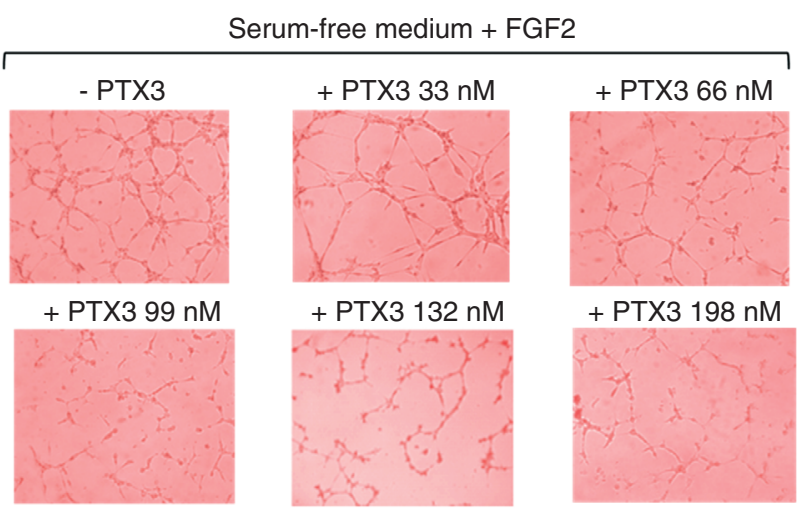

inhibition of vessel length PTX3 (nM)

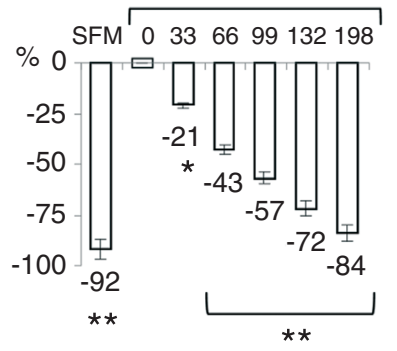

inhibition of branching points PTX3 (nM)

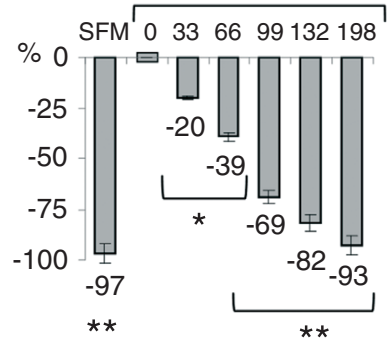

CAM assay

MM PC conditioned medium

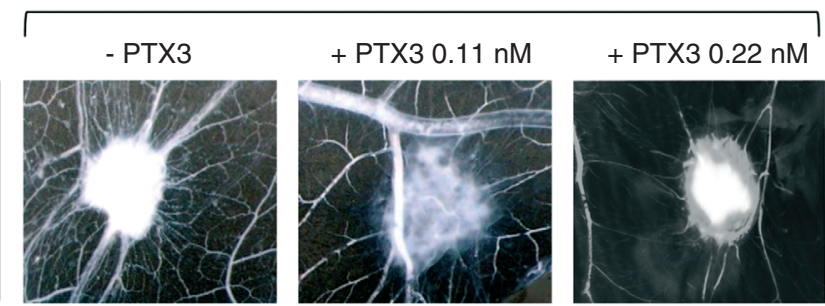

n. $=26 \pm 5$

n. $=6 \pm 2$

$\star \star$

Figure 4. PTX3 inhibits angiogenesis in Matrige ${ }^{\circledR}$ and chick embryo chorioallantoic membrane (CAM) assays. (A) MM endothelial cells (ECs) from 18 patients were seeded on Matrigel ${ }^{\circledR}$ in serum-free medium (SFM) and SFM supplemented with FGF2 (0.58 nM) and PTX3 doses. After an $8 \mathrm{~h}$ incubation, inhibition of vessel mesh areas, length, and branching points was examined by image analysis software. ${ }^{*} p<0.03,{ }^{* *} p<0.01$; Wilcoxon signed-rank test. Matrigel ${ }^{\circledR}$ pictures from a representative patient are shown. (B) CAMs were treated with sponges loaded with SFM (negative control: physiological angiogenesis) or MM plasma cell (PC) conditioned media from $10 \mathrm{MM}$ patients in the absence or presence of 0.11 and $0.22 \mathrm{nM}$ PTX3. Angiogenesis was measured and is expressed as mean \pm SD vessel counts. ${ }^{*} p<0.03,{ }^{* *} p<0.01$; Wilcoxon signed-rank test.

affected the viability of MM cells and their adhesion to BMSCs. Flow cytometry analysis of PTX3treated PC lines demonstrated that direct treatment with PTX3 reduced only marginally the viability of MM PCs. Specifically, apoptosis in untreated control RPMI8226 cells was $12 \%$ (Figure 6A, picture I) and exposure to 66 and $132 \mathrm{nM}$ PTX3 caused only a limited increase in the apoptotic index $(27 \%$ and $29 \%$, respectively; Figure 6A, II and III). On this basis, to assess whether PTX3 may affect MM PC viability indirectly, ie via inhibition of ECs and FBs, RPMI8226 cells were co-cultured with these BMSCs, exposed to PTX3, and assessed for apoptosis. In a first set of experiments, RPMI8226 cells were co-cultured with MM ECs and FBs separated by a transwell insert to prevent cell-cell contact in the absence or in the presence of PTX3. Under these experimental conditions, we observed no changes in the apoptotic index of co-cultured RPMI8226 cells compared with the cell line alone (Figure 6A, IV-VI). On the contrary, when RPMI8226 cells were co-cultured without the transwell insert to allow direct cell-cell contact with BMSCs, 66 and $132 \mathrm{nM}$ PTX3 were able to induce apoptosis in PCs by increasing the RPMI8226 cell apoptotic index to $38 \%$ and $62 \%$, respectively (Figure 6A, VII-IX). A similar behaviour was observed in the U266 cell line 
A
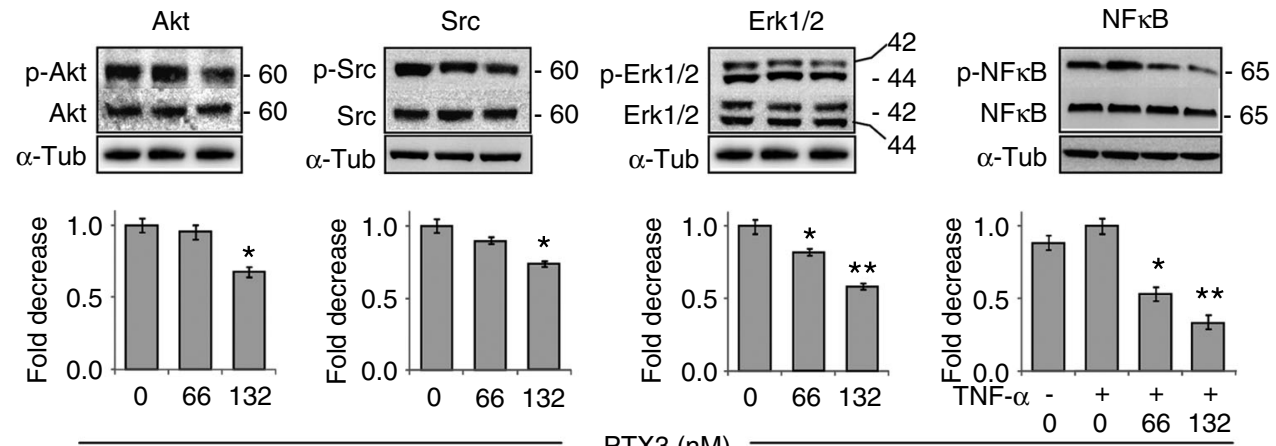

B Western blot of total lysates of MM fibroblasts
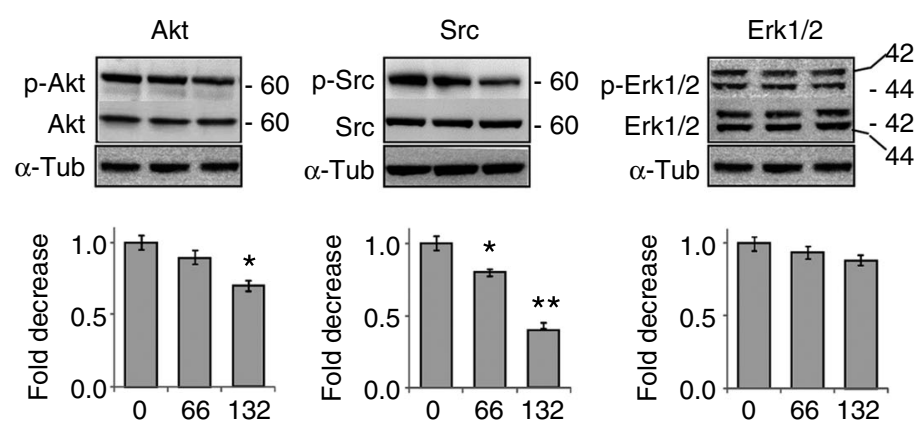

PTX3 (nM)
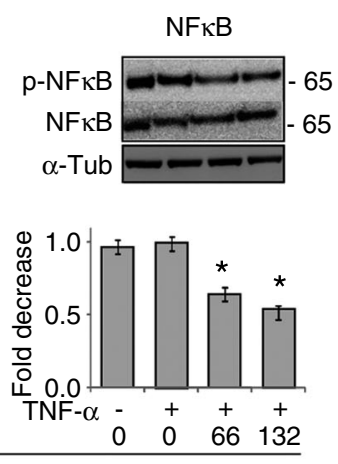

C

ELISA of conditioned medium of:

MM endothelial cells

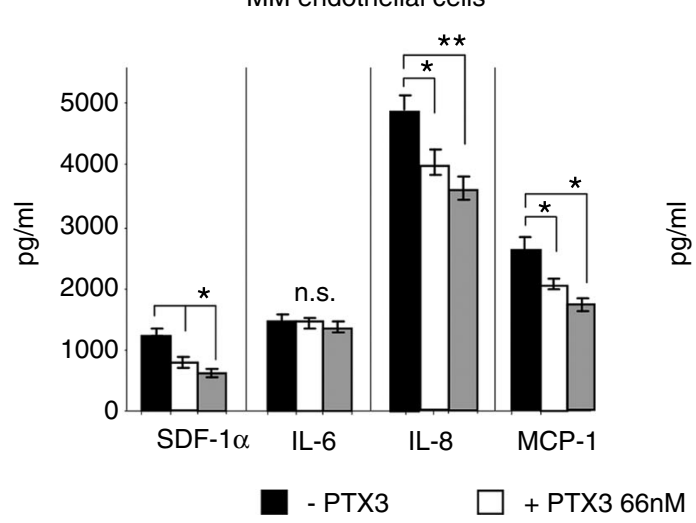

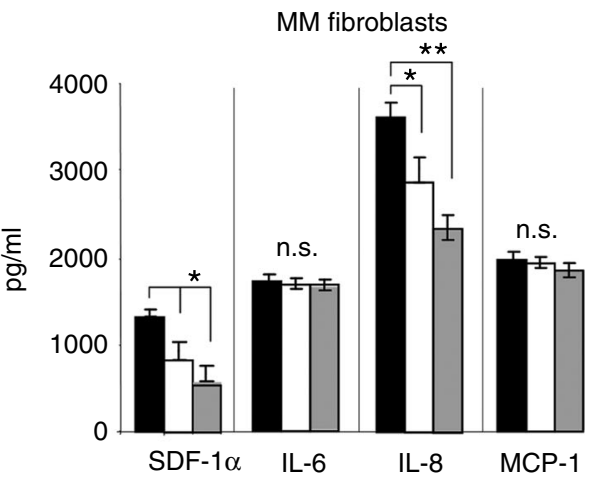

$\square+$ PTX3 132nM

Figure 5. PTX3 modulates FGF2-signalling pathway and cytokine secretion by MM endothelial cells (ECs) and fibroblasts (FBs). (A, B) MM ECs (A) and FBs (B) purified from the BM of 15 patients were treated for 15 min with PTX3 $(0,66$, and $132 \mathrm{nM})$ in serum-free medium (SFM) supplemented with FGF2 (0.58 nM) and studied for expression of Akt, phospho (p)-Akt, Src, p-Src, Erk1/2, and p-Erk1/2 by western blot. NFKB and p-NFKB were evaluated by treating MM ECs and FBs for 25 min with PTX3 $(0,66$, and $132 \mathrm{nM})$ in serum-free medium (SFM) supplemented with FGF2 $(0.58 \mathrm{nM})$ and then treated with TNF- $\alpha(10 \mathrm{ng} / \mathrm{ml})$ for $10 \mathrm{~min}$. $\alpha$-tubulin = loading control. Densitometry values are shown as fold decrease compared with the control. Gels of cells from a representative patient are shown. (C) Measurement of the indicated cytokines in the conditioned media of MM ECs and FBs was performed by ELISA. ${ }^{*} p<0.03,{ }^{* *} p<0.01$; Wilcoxon signed-rank test. SDF-1 $\alpha$, stromal cell derived factor-1 alpha; IL-6, interleukin-6; IL-8, interleukin-8; MCP-1, monocyte chemotactic protein-1.

(data not shown). The effect of PTX3 on MM ECs and FBs co-cultured with RPMI8226 cells (with or without transwell) was also analysed. As shown in Figure 6A, $\mathrm{X}-\mathrm{XII}$, exposure to 66 and $132 \mathrm{nM}$ PTX3 induced a significant increase in the apoptotic index $(36 \%$ and $57 \%$, respectively) that was not modified either in the presence or in the absence of a transwell insert (data not shown). In order to confirm the PTX3 effect also on primary MM cells, flow cytometry analysis of PTX3treated fresh PCs alone or co-cultured with paired MM ECs and FBs was performed (Supplementary Figure 4). The basal apoptosis percentage of PCs was higher than that of cell lines due to their intrinsic frailty, and this was prevented by co-culture with stromal cells $(27 \%$ versus $11 \%$ apoptotic cells, Supplementary Figure 4, I and IV). 66 and 132 nM PTX3 were able to increase 


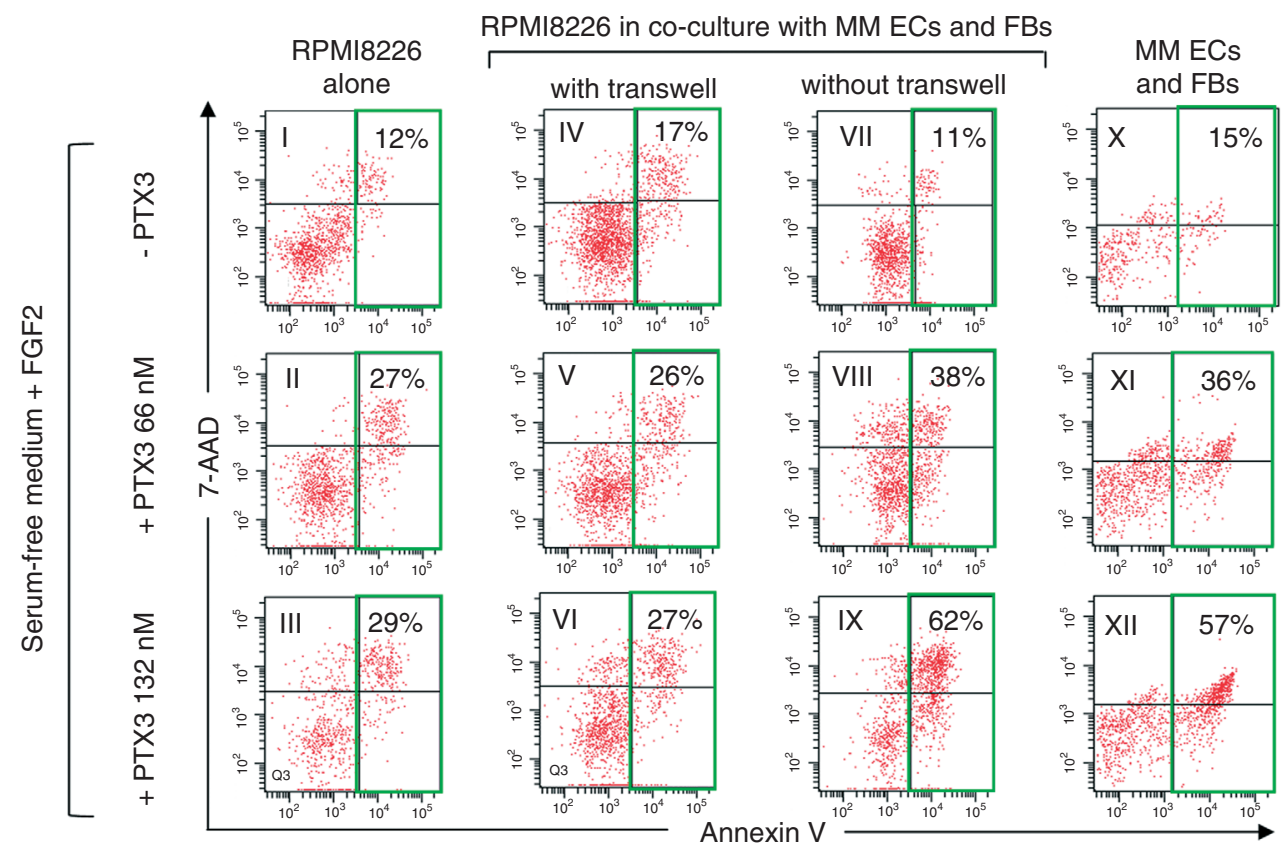

B MM endothelial cells

RPMI8226 adhesion on:
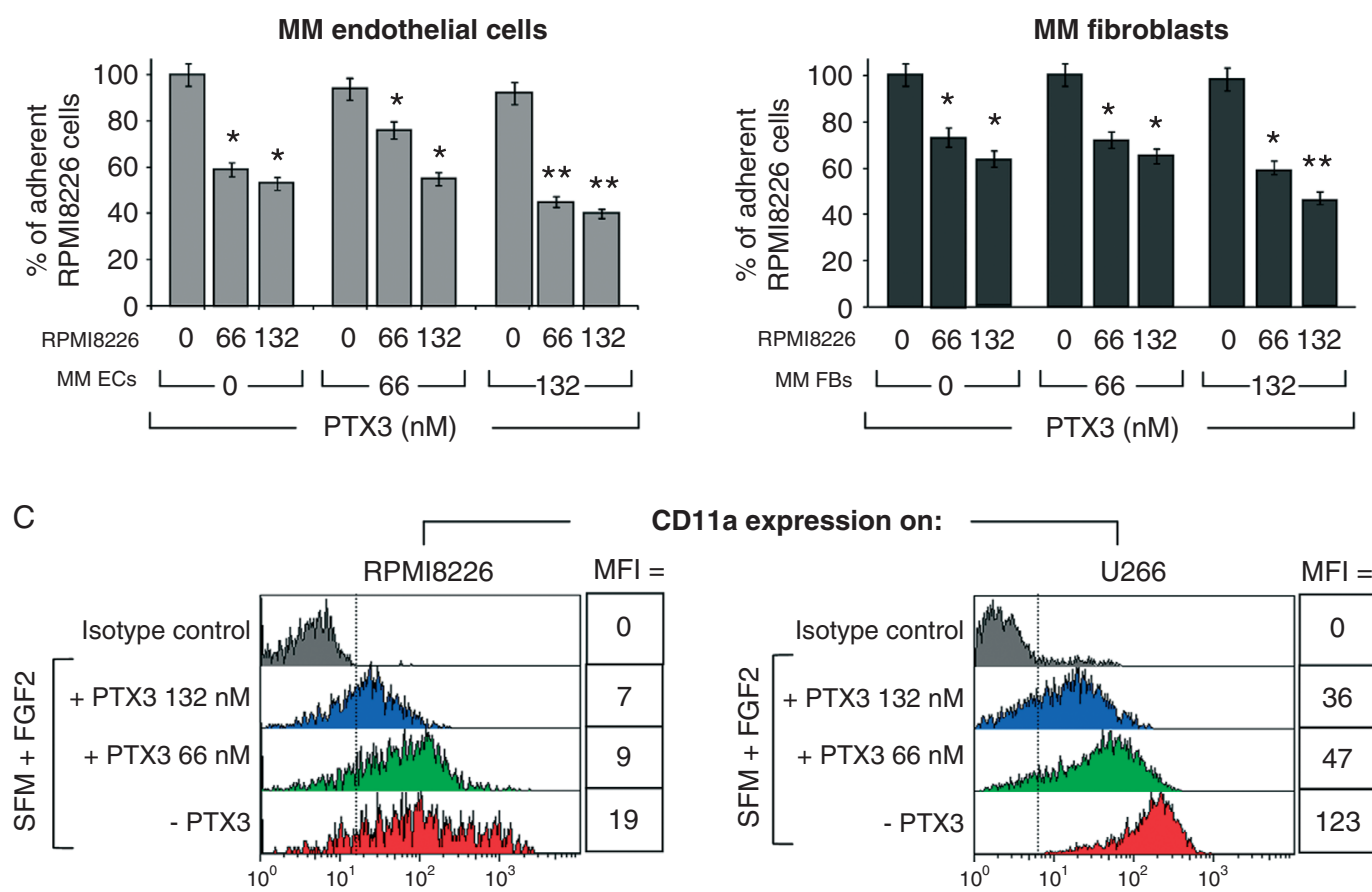

CD11a expression on:

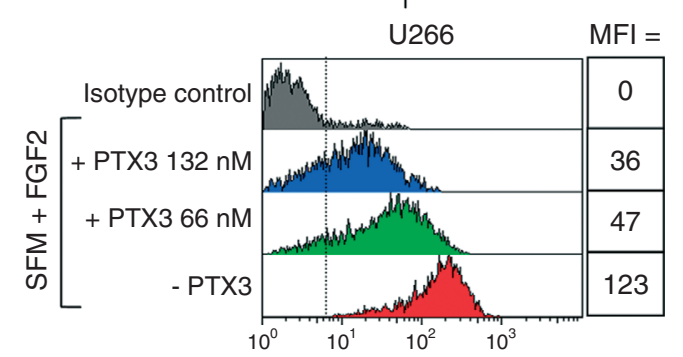

Figure 6. PTX3 affects plasma cell (PC), endothelial cell (EC), and fibroblast (FB) apoptosis and PC adhesion to MM ECs and FBs. (A) Apoptosis of RPMI8226 and MM ECs and FBs. RPMI8226 cells alone (I-III), RPMI8226 co-cultured with ECs and FBs (IV-IX), and ECs and FBs alone (X-XII) purified from 16 MM patients were seeded in serum-free medium (SFM) and SFM supplemented with $0.58 \mathrm{nM}$ FGF2 and PTX3 $(0,66$, and $132 \mathrm{nM})$. Co-cultures were performed with or without a transwell insert. After $24 \mathrm{~h}$ of PTX3 treatment, apoptosis of RPMI8226 cells and of MM ECs and FBs (X-XII) was analysed by flow cytometry using Annexin-V/7-AAD. Green rectangles show early (bottom box) and late (top box) apoptotic cells. Dot plots of BM ECs and FBs from a representative MM patient are shown. (B) Adhesion assay of RPMI8226 cells on MM ECs and FBs. RPMI8226 cells and MM ECs and FBs were treated for $24 \mathrm{~h}$ with PTX3 $(0,66$, and 132 nM) in SFM added with 0.58 nM FGF2. RPMI8226 cells, labelled with Calcein AM Fluorescent Dye, were seeded onto 96-well plates coated with MM ECs and FBs for $2 \mathrm{~h}$ to allow adhesion. Adherent PCs were analysed by a fluorescence assay. ${ }^{*} p<0.03,{ }^{* *} p<0.01$; Wilcoxon signed-rank test. (C) CD11a (LFA-1) expression on RPMI8226 and U266 cells cultured for $24 \mathrm{~h}$ in SFM supplemented with FGF2 (0.58 nM) and PTX3 $(0,66$, and $132 \mathrm{nM})$ was evaluated by flow cytometry. MFI = mean fluorescence intensity. 
apoptosis by $36 \%$ and $49 \%$, respectively (Supplementary Figure 4, II and III). When PCs were co-cultured with paired stromal cells, PTX3 produced a higher apoptotic effect: $53 \%$ and $68 \%$, respectively (Supplementary Figure 4, V and VI).

We then examined whether PTX3 can affect MM PC adhesion to ECs and FBs. As shown in Figure 6B, adhesion of PTX3-treated RPMI8226 was markedly inhibited in a dose-dependent fashion on both untreated and treated MM ECs and FBs. On the contrary, no effect was observed when untreated RPMI8226 cells were seeded on treated MM ECs and FBs. The loss of adhesive capability was mainly due to the downregulation of lymphocyte function-associated antigen1 (LFA-1/CD11a) expression on both PC lines. In fact, flow cytometry analysis showed that treatment of RPMI8226 and U266 cell lines inhibits the percentage of $\mathrm{CD}_{11 \mathrm{a}^{+}}$cells as well as their mean fluorescence intensity (Figure 6C). The expression of other adhesive molecules, ie CD54, CD49d, CD49e, and CD29, on PC lines (Supplementary Figure 5) and on MM ECs and FBs (Supplementary Figure 6) did not vary significantly.

These data suggest that PTX3 drives both a direct apoptotic effect on MM ECs and FBs and an indirect apoptotic effect on PCs through its capability to inhibit $\mathrm{PC} / \mathrm{BMSC}$ adhesion.

\section{Discussion}

The superfamily of FGFs/FGFRs controls a wide range of biological functions [27]. Specifically, FGF2 is a pleiotropic growth factor that acts in an autocrine and paracrine fashion on the tumour, stroma, and inflammatory cells of several neoplastic diseases [26] including MM [27]. High FGF2 levels are required for vascular sprouting and integrity, while inhibition of FGFR signalling causes angiostasis and vessel disassembly [28]. FGF2 also favours VEGF to exert its pro-angiogenic effect on ECs [29]. In MM, the FGF2 levels are significantly higher in PC lysates of patients with active disease compared with those with non-active disease and MGUS, and inhibition of FGF2 suppresses the angiogenic potential of PCs, suggesting a key role for these cells in the angiogenic switch [25]. Overall, the data also indicate that FGF2 may directly or indirectly stimulate MM PC survival and proliferation and be considered a plausible therapeutic target [26].

PTX3 is a natural selective antagonist for a restricted number of FGF family members, inhibiting FGF2 but not FGF1 or FGF4 activity [13]. PTX3 levels increase under inflammatory conditions caused by infection, tissue damage, and cancer [30-32]. Accordingly, here we show that MM PCs, ECs, and FBs secrete increased levels of PTX3 as well as FGF2. Analysis of data as FGF2/PTX3 ratio demonstrated that in MM this ratio is shifted towards the FGF2 production according to the pro-angiogenic and inflammatory state of BM MM compared with BM MGUS and control subjects.

To elucidate the effects of PTX3 on PC/stroma cross-talk, we analysed BMSCs such as ECs and FBs as well as PCs from MM and MGUS patients for (i) responsiveness to exogenous PTX3 and (ii) changes in the downstream signalling of the FGF2/FGFR system upon exposure to PTX3. Overall, the study aimed at identifying and testing a potential anti-angiogenic and anti-PC role of PTX3 in MM that could suggest it as a potential therapeutic target. MM ECs were used as over-angiogenic ECs as opposed to MGUS ECs, control subjects' ECs, and HUVECs, the norm-angiogenic counterpart [4,19].

Our results demonstrate that PTX3 inhibits FGF2induced angiogenesis of MM ECs through its binding to FGF2 (Figure 4). It does not affect VEGF-dependent angiogenesis, since it is not able to interact with VEGF (Figure 3), in line with Rusnati et al's data on HUVECs and bovine aortic ECs [15]. We have also shown that PTX3 impacts some biological functions of ECs and FBs isolated from the BM of MM patients (Figure 2) by inhibiting their viability and FGF2induced migration. Indeed, we demonstrated that PTX3 effects were mediated by down-regulation of several FGF2-induced downstream signalling pathways, such as p-Akt, p-Erk1/2, p-Src, and p-NFkB (Figure 5). The effects of PTX3 on MGUS ECs and FBs were only marginal, suggesting that these cells are not addicted to FGF2/FGFR pathways.

Since the BM microenvironment plays a crucial role in PC proliferation, survival, and drugs resistance, we wondered whether PTX3 could indirectly hit PCs through its inhibitory activity on BMSCs. We found that PTX3 treatment is has as much negligible direct cytotoxicity on MM cell lines as much as on fresh PCs, but it induces significant PC apoptosis when these cells are directly co-cultured with MM ECs and FBs (Figure 6 and Supplementary Figure 4). It has been already demonstrated that FGF2 promotes the stromal cell derived factor-1 alpha $(\mathrm{SDF}-1 \alpha)$ production through overexpression of its transcript via the ERM transcription factor [33], and stimulates at least four to five times the monocyte chemotactic protein-1 (MCP1) production in bovine aortic endothelial cells [34]. Tentatively, we hypothesize that the cytotoxic effect of PTX3 may depend on its ability to (i) sequestrate FGF2 with consequent reduction of SDF-1 $\alpha$, MCP-1, and IL- 8 production by both ECs and FBs (Figure 4); (ii) down-regulate the expression of LFA-1 on MM PCs (Figure 6); and (iii) induce the apoptosis of MM ECs and FBs (Figure 6). It is thus plausible that PTX3 exerts an indirect BMSC-mediated effect on PCs, reducing their supply and chemotactic stimuli, impairing the adhesive PC capability, and causing their detachment from stromal ECs and FBs.

The BM microenvironment is the source of a complex pattern of signals in which PCs and BMSCs reciprocally communicate, resulting in a feeder for PCs. In this context, cytokines (such as FGF2, SDF-1 $\alpha$, IL-6, 
IL-8, and MCP-1) become fundamental since they act in an autocrine and paracrine fashion on PCs. Human MM cell lines (RPMI8226 and U266) did not show constitutive FGFR activation nor did they activate proliferation or survival after FGF2 stimulation, ruling out possible autocrine or paracrine FGF2-mediated loops in these cell lines.

In conclusion, we have shown a potent PTX3 antiangiogenic effect on MM ECs, due to its capability to bind to FGF2 [15], and a direct impact on FGF2induced biological activities on MM FBs which also support PC growth. PTX3 is cytotoxic on MM cells by inhibiting the activities of ECs and FBs including cytokine production, and causing loss of adhesive PC capability to these cells. Thus, PTX3 may play an important role in inhibiting the cross-talk between these BMSCs and PCs in patients with MM.

Our data also suggest that at the angiogenic switch MM ECs acquire high expression/activation of the FGF2 signalling, which contributes to pathological BM angiogenesis and overcomes the inhibitory effect of the PTX3 autocrine loops usually activated to selflimit physiological angiogenesis in a normal subject or MGUS patient. Kumar et al [35] hypothesized an inhibitory (anti-angiogenic) activity in MGUS patients that is lost with the angiogenic switch, ie with transition to MM. We suggest that the expression of PTX3 by MGUS ECs may be a self-limiting feature of MGUS angiogenesis, which is overcome by high expression of FGF2 at the angiogenic switch when MGUS ECs are driven towards an MM EC over-angiogenic phenotype by PCs and BMSCs. The exposure of MM ECs to exogenous PTX3 appears to re-equilibrate the abnormal excess of endogenous FGF2 and leads them to a norm-angiogenic state like MGUS ECs, control ECs, and HUVECs.

Indeed, FGF2-induced proliferation, migration, and angiogenesis on the Matrigel ${ }^{\circledR}$ surface and in the CAM were also significantly inhibited by recombinant PTX3. Hence, recombinant PTX3, acting as an agent aimed at blocking excessive FGF2 activity and re-establishing a physiological FGF2/PTX3 ratio, may be a possible future area of research in the field of anti-angiogenesis in MM.

This study shows limitations that we wish to cover. We will evaluate the PTX3 effects in MM patients in relation to the D\&S stage. Also, we have planned to apply PTX3 to an in vivo model other than CAM, ie the NOD-scid MM mouse, in order to study better its anti-MM and anti-angiogenic effects. Finally, we will evaluate the activity of PTX3 in conjunction with the new anti-MM drugs, such as bortezomib, thalidomide, and lenalidomide, to assess its potential additive or synergistic effects in inhibiting plasma cell/stromal cell cross-talk.

\section{Acknowledgment}

This work was supported by Associazione Italiana per la Ricerca sul Cancro (AIRC), Investigator Grant and Special Program Molecular Clinical Oncology $5 \times 1000$ n. 9965, Milan, to AV, and the EU Multiple Myeloma Program FP7 OVER-MyR HEALTH.2011. 2.4.1-2 to AV, and EU FPT7 (2007-2013) under grant agreement No 278570 to DR, and grants from MIUR, AIRC (grant No 10396), Fondazione Berlucchi, CARIPLO (grant 2008-2264 and NOBEL Project) to MP. AB was supported by a studentship from Federazione Italiana per la Ricerca sul Cancro (FIRC), Milan, Italy. AM was supported by European Research Council (Project HIIS) and Special Program Molecular Clinical Oncology $5 \times 1000$ n. 9962 'INNATE IMMUNITY IN CANCER (IIC)'.

\section{Abbreviations}

$\begin{array}{ll}\text { 7-AAD } & \text { 7-amino-actinomycin } \\ \text { BM } & \text { bone marrow } \\ \text { BMSC } & \text { bone marrow stromal cell } \\ \text { CAM } & \text { chick embryo chorioallantoic membrane } \\ \text { CFSE } & \text { carboxyfluorescein N-succinimidyl ester } \\ \text { CM } & \text { conditioned medium } \\ \text { EC } & \text { endothelial cell } \\ \text { FB } & \text { fibroblast } \\ \text { FGF2 } & \text { fibroblast growth factor-2 } \\ \text { MCP-1 } & \text { monocyte chemotactic protein-1 } \\ \text { MGUS } & \text { monoclonal gammopathy of undetermined } \\ & \text { significance } \\ \text { MM } & \text { multiple myeloma } \\ \text { PC } & \text { plasma cells } \\ \text { PTX3 } & \text { pentraxin 3 } \\ \text { SDF-1 } \alpha & \text { stromal cell derived factor-1 alpha } \\ \text { SFM } & \text { serum-free medium } \\ \text { VEGF } & \text { vascular endothelial growth factor }\end{array}$

\section{Author contribution statement}

$\mathrm{AB}$ and $\mathrm{MM}$ planned and performed experiments and wrote the manuscript. RR, IM, ADL, and SB performed experiments. MAF performed experiments and wrote the manuscript. BB contributed material and commented on the manuscript. PD, EA, DD, GS, PC, VP, and BR contributed material. DR and MP planned and supervised the experiments. AM and FD commented on the manuscript. AV supervised the experiments, provided financing, and wrote the manuscript.

\section{References}

1. Raab MS, Podar K, Breitkreutz I, et al. Multiple myeloma. Lancet 2009; 374: 324-339.

2. Podar K, Tai YT, Hideshima T, et al. Emerging therapies for multiple myeloma. Expert Opin Emerging Drugs 2009; 14: 99-127.

3. Vacca A, Ribatti D. Bone marrow angiogenesis in multiple myeloma. Leukemia 2006; 20: 193-199. 
4. Vacca A, Ria R, Semeraro F, et al. Endothelial cells in the bone marrow of patients with multiple myeloma. Blood 2003; 102: 3340-3348.

5. Italiano Jr JE, Richardson JL. Angiogenesis is regulated by novel mechanism: pro- and anti-angiogenic proteins are organized into separate platelet alpha granules and differentially released. Blood 2008; 111: 1227-1233.

6. Seghezzi G, Patel S, Ren CJ, et al. Fibroblast growth factor-2 (FGF2) induces vascular endothelial factor (VEGF) expression in the endothelial cells of forming capillaries: an autocrine mechanism contributing to angiogenesis. J Cell Biol 1998; 141: 1659-1673.

7. Jakob C, Sterz J, Zavrski I, et al. Angiogenesis in multiple myeloma. Eur J Cancer 2006; 42: 1581-1590.

8. Alessi P, Leali D, Camozzi M, et al. Anti-FGF2 approaches as a strategy to compensate resistance to anti-VEGF therapy: longpentraxin 3 as a novel antiangiogenic FGF2-antagonist. Eur Cytokine Netw 2009; 20: 225-234.

9. Kanazawa S, Fujiwara T, Matsuzaki S, et al. bFGF regulates PI3kinase-Rac1-JNK pathway and promotes fibroblast migration in wound healing. PLoS One 2010; 5: e12228.

10. Bottazzi B, Vouret-Cravari V, Bastone A, et al. Multimer formation and ligand recognition by long pentraxin PTX3. Similarities and differences with the short pentraxins C-reactive protein and serum amyloid P component. J Biol Chem 1997; 272: 32817-32823

11. Lee GW, Lee TH, Vilcek J. TSG-14, a tumor necrosis factor- and IL-1-inducible protein, is a novel member of the pentraxin family of acute phase proteins. J Immunol 1993; 150: 1804-1812.

12. Mantovani A, Garlanda C, Bottazzi B. Pentraxin 3, a nonredundant soluble pattern recognition receptor involved in innate immunity. Vaccine 2003; 21: 43-47.

13. Presta M, Camozzi M, Salvatori G, et al. Role of the soluble pattern recognition receptor PTX3 in vascular biology. J Cell Mol Med 2007; 11: 723-738.

14. Camozzi M, Rusnati M, Bugatti A, et al. Identification of an antiangiogenic FGF2-binding site in $\mathrm{N}$ terminus of the soluble pattern recognition receptor PTX3. J Biol Chem 2006; 281: $22605-22613$

15. Rusnati M, Camozzi M, Moroni E, et al. Selective recognition of fibroblast growth factor-2 by the long pentraxin PTX3 inhibits angiogenesis. Blood 2004; 104: 92-99.

16. Leali D, Bianchi R, Bugatti A, et al . Fibroblast growth factor 2antagonist activity of a long-pentraxin 3-derived antiangiogenic pentapeptide. J Cell Mol Med 2010; 14: 2109-2121.

17. Introna M, Alles VV, Castellano M, et al. Cloning of mouse ptx3, a new member of the pentraxin gene family expressed at extrahepatic sites. Blood 1996; 87: 1862-1872.

18. Alles VV, Bottazzi B, Peri G, et al. Inducible expression of PTX3, a new member of the pentraxin gene family, in human mononuclear phagocytes. Blood 1994; 84: 3483-3493.

19. Berardi S, Caivano A, Ria R, et al. Four proteins governing overangiogenic endothelial cell phenotype in patients with multiple myeloma are plausible therapeutic targets. Oncogene 2012; 31: $2258-2269$
20. Vacca A, Scavelli C, Montefusco V, et al. Thalidomide downregulates angiogenic genes in bone marrow endothelial cells of patients with active multiple myeloma. J Clin Oncol 2005; 23: 5334-5346.

21. De Luisi A, Ferrucci A, Coluccia AML, et al. Lenalidomide restrains mobility and overangiogenic potential of bone marrow endothelial cells in patients with acute multiple myeloma. Clin Cancer Res 2011; 17: 1935-1946.

22. Guidolin D, Vacca A, Nussdorfer GG, et al. A new image analysis method based on topological and fractal parameters to evaluate the angiostatic activity of docetaxel by using the Matrigel ${ }^{\circledR}$ assay in vitro. Microvasc Res 2004; 67: 117-124.

23. Ribatti D, Nico B, Vacca A, et al. The gelatin spongechorioallantoic membrane assay. Nature Protoc 2006; 1: 85-91.

24. Lambert KE, Huang H, Mythreye K, et al. The type III transforming growth factor- $\beta$ receptor inhibits proliferation, migration, and adhesion in human myeloma cells. Mol Biol Cell 2011; 22: 1463-1472.

25. Vacca A, Ribatti D, Presta M, et al. Bone marrow neovascularization, plasma cell angiogenic potential, and matrix metalloproteinase-2 secretion parallel progression of human multiple myeloma. Blood 1999; 93: 3064-3073.

26. Ribatti D, Nico B, Vacca A. Importance of the bone marrow microenvironment in inducing the angiogenic response in multiple myeloma. Oncogene 2006; 25: 4257-4266.

27. Beenken A, Mohammadi M. The FGF family: biology, pathophysiology and therapy. Nature Rev Drug Discov 2009; 8: 235-253.

28. Murakami M, Nguyen LT, Zhuang ZW, et al. The FGF system has a key role in regulating vascular integrity. J Clin Invest 2008; 118 3355-3366.

29. Murakami M, Nguyen LT, Hatanaka K, et al. FGF-dependent regulation of VEGF receptor 2 expression in mice. J Clin Invest 2011; 121: $2668-2678$.

30. Mantovani A, Garlanda C, Doni A, et al. Pentraxins in innate immunity: from $\mathrm{C}$-reactive protein to the long pentraxin PTX3. J Clin Immunol 2008; 28: 1-13.

31. Vänskä M, Koivula I, Hämäläinen S, et al. High pentraxin 3 level predicts septic shock and bacteremia at the onset of febrile neutropenia after intensive chemotherapy of hematologic patients. Haematologica 2011; 96: 1385-1389.

32. Diamandis EP, Goodglick L, Planque C, et al. Pentraxin-3 is a novel biomarker of lung carcinoma. Clin Cancer Res 2011; 17: 2395-2399.

33. Yoon KA, Chae YM, Cho JY. FGF2 stimulates SDF-1 expression through the Erm transcription factor in Sertoli cells. J Cell Physiol 2009; 220: 245-256.

34. Wempe F, Lindner V, Augustin HG. Basic fibroblast growth factor (bFGF) regulates the expression of the $\mathrm{CC}$ chemokine monocyte chemoattractant protein-1 (MCP-1) in autocrine-activated endothelial cells. Arterioscler Thromb Vasc Biol 1997; 17: 2471-2478.

35. Kumar S, Witzig TE, Timm M, et al. Bone marrow angiogenic ability and expression of angiogenic cytokines in myeloma: evidence favoring loss of marrow angiogenesis inhibitory activity with disease progression. Blood 2004; 104: 1159-1165. 


\section{SUPPORTING INFORMATION ON THE INTERNET}

The following supporting information may be found in the online version of this article.

\section{Supplementary materials and methods}

Figure S1. PTX3 effects on MGUS and normal endothelial cell (EC) functional activities.

Figure S2. PTX3 effects on MGUS and normal fibroblast (FB) functional activities.

Figure S3. PTX3 effects on MGUS and normal endothelial cell (EC) angiogenesis.

Figure S4. PTX3 affects apoptosis of fresh MM plasma cells (PCs).

Figure S5. Expression of the indicated adhesion molecules on RPMI8226 and U266 cells upon PTX3 treatment.

Figure S6. Expression of the indicated adhesion molecules on MM ECs and FBs upon PTX3 treatment. 